hep-th/0608213

KUNS-2038

\title{
AdS/CFT and Strong Subadditivity of Entanglement Entropy
}

\author{
Tomoyoshi Hirata1 and Tadashi Takayanagi2 \\ Department of Physics, Kyoto University, Kyoto 606-8502, Japan
}

\begin{abstract}
Recently, a holographic computation of the entanglement entropy in conformal field theories has been proposed via the AdS/CFT correspondence. One of the most important properties of the entanglement entropy is known as the strong subadditivity. This requires that the entanglement entropy should be a concave function with respect to geometric parameters. It is a non-trivial check on the proposal to see if this property is indeed satisfied by the entropy computed holographically. In this paper we examine several examples which are defined by annuli or cusps, and confirm the strong subadditivity via direct calculations. Furthermore, we conjecture that Wilson loop correlators in strongly coupled gauge theories satisfy the same relation. We also discuss the relation between the holographic entanglement entropy and the Bousso bound.
\end{abstract}

August, 2006

1 e-mail:hirata@gauge.scphys.kyoto-u.ac.jp

2 e-mail:takayana@gauge.scphys.kyoto-u.ac.jp 


\section{Introduction}

In order to understand quantum gravity, we need to know the structure of its Hilbert space $\mathcal{H}_{\text {tot }}$ and the quantum state $|\Psi\rangle$ which describes a given background. In this setup one of the most basic quantities is considered to be the entanglement entropy. Let us divide the total Hilbert space into a direct product of two subspaces $\mathcal{H}_{\text {tot }}=\mathcal{H}_{A} \otimes \mathcal{H}_{B}$. The entanglement entropy is defined as the von Neumann entropy when we trace out the subspace $\mathcal{H}_{B}$. This measures the information loss accompanied with this smearing process. The entropy depends only on the total density matrix $\rho_{t o t}=|\Psi\rangle\langle\Psi|$ and the structure of the Hilbert space. Therefore it is a universal and basic quantity to characterize the quantum state as we do not have to make explicit the details of the theory such as matter contents and so on.

The modern understanding of holography, especially in the AdS/CFT correspondence [1], tells us that the Hilbert space $\mathcal{H}_{\text {tot }}$ of the gravity theory is actually equivalent to the Hilbert space of a certain quantum field theory (QFT) that lives on the boundary of the spacetime. In AdS/CFT, the QFT becomes a conformal field theory (CFT) and this is the setup we consider in this paper mainly.

In QFTs, we have two Hilbert subspaces by dividing the total space manifold into two submanifolds $A$ and $B$. The entanglement entropy is defined geometrically by tracing out the states which live on the submanifold $A$ [2]. The holography argues that the Hilbert space of its dual gravity theory can also be written as a direct product as is necessary to define the entanglement entropy. Moreover, we expect that the entanglement entropy computed in the dual field theory should be the same as the one in the gravity theory.

Recently, a holographic computation of the entanglement entropy is proposed in [3] [4], based on the AdS/CFT correspondence [1]. There, the entropy is calculated by replacing the horizon area in the Bekenstein-Hawking formula with the area of minimal surface in AdS space whose boundary is the same as that of the submanifold $A$. This offers us a powerful way to calculate the entanglement entropy; otherwise it involves the complicated quantum analysis, which is very hard except two dimensional CFTs [5]. This relation has been successfully applied to black holes in brane-world [6] [7] and de-Sitter spaces [8] (refer to [9] and [10] for earlier discussions). A slightly heuristic proof of this 
proposal has been given in [11] based on the first principle of AdS/CFT correspondence [12]. Moreover, as we will point out in this paper, the above holographic computation of entanglement entropy is closely related to the covariant entropy bound known as the Bousso bound [13. We will argue that the holographic computation saturates this bound. This consideration makes clear the following basic question in AdS/CFT correspondence: what part of the AdS space encodes the information of the boundary CFT included in a specific region?

In this paper we would like to explore this proposal and to find further evidences from a different viewpoint. One of the most important properties which are satisfied by the entanglement entropy is called the strong subadditivity [14. This represents a sort of irreversibility of entropy and is considered to be the strongest condition of entanglement entropy. Indeed, it has rigorously been shown that the strong subadditivity with several other more obvious conditions characterizes the von-Neumann entropy. Moreover, it is known that the entropic analogue of Zamolodchikov's $c$-theorem can be derived from the strong subadditivity in 2D QFTs [15] 16].

The main purpose of this paper is to check if this condition is satisfied in the holographic calculations in several explicit examples. In particular we consider the case where the submanifold $A$ is given by an annulus, and also the case where its boundary $\partial A$ is a line with a cusp singularity. The latter examples in the $(2+1) \mathrm{D}$ free scalar field theory were discussed in [17] quite recently (refer to [18] for a general discussion and also to [19] for an analysis at quantum critical point with a conformal invariant ground state wavefunction). Our analysis corresponds to the result in the strongly coupled supersymmetric gauge theories of the same dimension. The strong subadditivity requires that a specific term in the entropy should be a concave function ${ }^{3}$ when we change the value of the geometrical quantity such as the ratio of the radius in the annulus and the deficit angle of a cusp. Indeed, in all our examples we will find that the strong subadditivity is satisfied. This provides an additional evidence of the proposed holographic derivation of the entanglement entropy. We can also regard it as an evidence of the AdS/CFT correspondence itself.

3 As usual, we call a function $f$ is concave (or convex) if $f^{\prime \prime} \leq 0$ (or $f^{\prime \prime} \geq 0$ ). Even though the specific terms in the entropy itself are concave, we will also encounter convex functions because we sometimes flip the sign of the terms. 
At the same time, as a bonus, we will notice the similarity between the holographic calculation of the entanglement entropy [3] and the well-known evaluation of the Wilson loops expectation values in $\mathrm{AdS} / \mathrm{CFT}$ [20]. In particular, we can easily find that the entanglement entropy in a $(2+1)$ dimensional CFT is equivalent to the Wilson loop in a $(3+1)$ dimensional CFT defined by a strongly coupled gauge theory. Thus we conjecture that the strong subadditivity relation is also true for strongly coupled gauge theories.

This paper is organized as follows. In section two, we review the basic definition and properties of the entanglement entropy such as the strong subadditivity. In section three, we apply the strong subadditivity to various examples of the entanglement entropy in CFTs. In section four, we explain the holographic computation of the entanglement entropy via the AdS/CFT. We also relate it to the covariant entropy bound (so called the Bousso bound). In section five, we examine specific examples of the holographic entanglement entropy and check if it satisfies the strong subadditivity. In section six, we summarize conclusions.

\section{Entanglement Entropy and Strong Subadditivity}

\subsection{Definition of Entanglement Entropy}

Consider a quantum mechanical system with many degrees of freedom such as spin chains. More generally, we can consider arbitrary lattice models or quantum field theories (QFTs) including conformal field theories (CFTs). We put the system at zero temperature and the total quantum system is described by the pure ground state $|\Psi\rangle$. We assume no degeneracy of the ground state. Then, the density matrix is that of the pure state

$$
\rho_{t o t}=|\Psi\rangle\langle\Psi|
$$

The von Neumann entropy of the total system is clearly zero $S_{t o t}=-\operatorname{tr} \rho_{t o t} \log \rho_{t o t}=0$.

4 The entanglement entropy is generally divergent in continuum theories. Therefore usually we assume an ultraviolet cutoff $a$ to regulate the quantum field theory. In this sense, strictly speaking, the entropy should always be defined in the regularized lattice version of a given quantum field theories. Below we assume that this is just a technical issue and that we can always have such a regularization. 
Next we divide the total system into two subsystems $A$ and $B$. In the spin chain example, we artificially cut off the chain at some point and divide the lattice points into two groups. Notice that physically we do not do anything to the system and the cutting procedure is an imaginary process. Accordingly the total Hilbert space can be written as a direct product of two spaces $\mathcal{H}_{\text {tot }}=\mathcal{H}_{A} \otimes \mathcal{H}_{B}$ corresponding to those of subsystems $A$ and $B$. Let $\mathcal{O}_{A}$ be an operator which acts non-trivially only on $A$. Then its expectation value is

$$
\left\langle\mathcal{O}_{A}\right\rangle=\operatorname{tr} \mathcal{O}_{A} \cdot \rho_{\text {tot }}=\operatorname{tr}_{A} \mathcal{O}_{A} \cdot \rho_{A}
$$

where the $\operatorname{trace} \operatorname{tr}_{A}$ is taken only over the Hilbert space $\mathcal{H}_{A}$. Here we defined the reduced density matrix $\rho_{A}$ defined by

$$
\rho_{A}=\operatorname{tr}_{B} \rho_{t o t},
$$

by tracing out the Hilbert space $\mathcal{H}_{B}$. Thus the observer who is only accessible to the subsystem $A$ feels as if the total system were described by the reduced density matrix $\rho_{A}$.

Then we define the entanglement entropy of the subsystem $A$ as the von Neumann entropy of the reduced density matrix $\rho_{A}$

$$
S_{A}=-\operatorname{tr}_{A} \rho_{A} \log \rho_{A}
$$

This entropy measures the amount of information lost by tracing out (or smearing) the subsystem $B$.

It is also possible to define the entanglement entropy $S_{A}(\beta)$ at finite temperature

$T=\beta^{-1}$. This can be done just by replacing (2.1) with the thermal one $\rho_{\text {thermal }}=e^{-\beta H}$, where $H$ is the total Hamiltonian. When $A$ is the total system, $S_{A}(\beta)$ is clearly the same as the thermal entropy.

\subsection{Strong Subadditivity}

The entanglement entropy enjoys several useful properties. When the system is at zero temperature (i.e. pure state), it is easy to show

$$
S_{A}=S_{B}
$$


which manifestly shows that the entropy is not extensive as opposed to the ordinary thermal entropy. This equality (2.5) is violated for a mixed state (e.g. finite temperature).

Below we assume that the state is not pure in general. When we start with two subsystems $A$ and $A^{\prime}$, we can show

$$
S_{A}+S_{A^{\prime}} \geq S_{A \cup A^{\prime}}
$$

This is called subadditivity. We find this relation intuitively clear since the information for the overlapped part $A \cap A^{\prime}$ is double counted in the left-hand side of (2.6). It is true even when $A \cap A^{\prime}$ is empty.

Actually, a stronger inequality is known to be satisfied (again we assume the mixed state generally). This is called the strong subadditivity [14] given by the following formula

$$
S_{A}+S_{A^{\prime}} \geq S_{A \cup A^{\prime}}+S_{A \cap A^{\prime}}
$$

Equally we can write this inequality as follows. Divide the subsystem $A$ into three parts $A_{1}, A_{2}$ and $A_{3}$ such that each of them does not intersect with each other, i.e.

$$
\mathcal{H}_{A}=\mathcal{H}_{A_{1}} \otimes \mathcal{H}_{A_{2}} \otimes \mathcal{H}_{A_{3}}
$$

then we can show

$$
S_{A_{1}+A_{2}+A_{3}}+S_{A_{2}} \leq S_{A_{1}+A_{2}}+S_{A_{2}+A_{3}}
$$

Notice that if there is no correlation between the three Hilbert spaces (i.e. $\rho_{\text {tot }}=\rho_{A_{1}} \otimes$ $\left.\rho_{A_{2}} \otimes \rho_{A_{3}}\right)$, the both sides in (2.9) become equal.

By multiplying an extra Hilbert space $A_{4}$ with $A_{1}, A_{2}$ and $A_{3}$ such that the system is pure with respect to the total Hilbert space $\mathcal{H}_{A_{\text {tot }}}=\mathcal{H}_{A_{1}} \otimes \mathcal{H}_{A_{2}} \otimes \mathcal{H}_{A_{3}} \otimes \mathcal{H}_{A_{4}}$, we can find another inequality equivalent to (2.9)

$$
S_{A_{1}}+S_{A_{3}} \leq S_{A_{1}+A_{2}}+S_{A_{2}+A_{3}}
$$

It is easy to see that the subadditivity (2.6) and the triangle inequality (Araki-Lieb inequality) [21]

$$
\left|S_{A_{1}}-S_{A_{2}}\right| \leq S_{A_{1}+A_{2}}
$$


can be derived from (i.e. weaker than) (2.9) and (2.10).

The strong subadditivity (2.7)(2.9) (2.10) is also satisfied for the classical entropy known as the Shannon entropy and in this case the proof is almost the same as the proof of the classical version of the subadditivity (2.6). However, the proof of the strong subadditivity for the quantum entropy (von Neumann entropy) is rather complicated. Indeed the original proof given by Lieb and Ruskai [14] is highly non-trivial.

To see an outlook of the proof for the strong subadditivity we begin with a definition of jointly concavity (we will follow the proof in [22]). Suppose $f(A, B)$ is a real-valued function of two matrices $A$ and $B$. The function $f$ is said to be jointly concave in $A$ and $B$ if

$$
f\left(\lambda A_{1}+(1-\lambda) A_{2}, \lambda B_{1}+(1-\lambda) B_{2}\right) \geq \lambda f\left(A_{1}, B_{1}\right)+(1-\lambda) f\left(A_{2}, B_{2}\right),
$$

for all $0 \leq \lambda \leq 1$

To prove the strong subadditivity, we firstly use the Lieb's theorem. Lieb's theorem states

$$
f(A, B) \equiv \operatorname{tr}\left(X^{\dagger} A^{t} X B^{1-t}\right)
$$

is jointly concave for any matrix $X$ and for all $0 \leq t \leq 1$ in positive matrices $A$ and $B$. Then Lieb's theorem implies the joint convexity of the relative entropy

$$
S(\rho \| \sigma) \equiv \operatorname{tr}(\rho \log \rho)-\operatorname{tr}(\rho \log \sigma) .
$$

in $\sigma$ and $\rho$, and the joint convexity of the relative entropy shows that the conditional entropy

$$
S(A \mid B) \equiv S_{A+B}-S_{B}
$$

is concave in $\rho_{A+B}$. Finally, strong subadditivity (2.7) (2.9) (2.10) follows from the concavity of the conditional entropy. More details of the proof and other properties of the entanglement entropy can be found in e.g. [22].

As is clear from the above proof, the strong subadditivity has a deep relationship with the concavity of entropy. It will become clear later that the strong subadditivity tells us the entropy is concave when we change the values of parameters that determine the subsystems. It is the strongest condition ever found for the von-Neumann entropy. Indeed, it has mathematically been shown that the strong subadditivity with several other more obvious conditions (such as the invariance under unitary transformations and the continuity with respect to the eigenvalues of $\rho_{t o t}$ ) characterizes the von-Neumann entropy [23]. 


\subsection{Entanglement Entropy in Relativistic Theories}

As we have explained, the entanglement entropy is defined by dividing the Hilbert space into two subspaces. In a $(d+1)$ dimensional quantum field theory, this can be done geometrically by specifying a $d$ dimensional submanifold $B$ embedded in the total $d$ dimensional space. Thus below we use the same symbols $A, B, \ldots$ to specify both the subsystem and the submanifold at the same time. We assume that the spacetime is simply given by the flat space $R^{1, d}$ just for simplicity.

In the relativistic theory we have to take care of the Lorentz invariance. We can take the space-like surface at fixed time $t=t_{0}$ as a Cauchy surface and divide it into $A$ and $B$. This manifestly divides the total Hilbert space $\mathcal{H}_{\text {tot }}$ into two subspaces $\mathcal{H}_{A}$ and $\mathcal{H}_{B}$ such that $\mathcal{H}=\mathcal{H}_{A} \otimes \mathcal{H}_{B}$. The entanglement entropy $S_{A}$ is define by taking the trace over states living on the submanifold $B$.

However, we can take other Cauchy surfaces to realize the same partition of the Hilbert space. Consider the surface $B^{\prime}$ instead of $B$ in Fig.1. This leads to the same Hilbert subspace i.e. $\mathcal{H}_{B^{\prime}}=\mathcal{H}_{B}$ because the physics on $B^{\prime}$ is completely determined if we

fix the initial condition on $B$. The information on $B$ determines $D^{+}(B)$, which is called the future domain of dependence. The boundary of $D^{+}(B)$ is the (future) Cauchy horizon $H^{+}(B)$. Therefore, the Hilbert subspace $\mathcal{H}_{B}$ is specified if we choose the Cauchy horizon $H^{+}(B)$ [16].

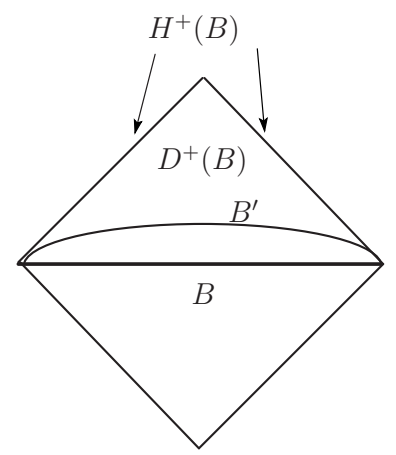

Fig. 1: The subspaces $B$ and $B^{\prime}$ of the Cauchy surfaces define the same Hilbert space $\mathcal{H}_{B^{\prime}}=\mathcal{H}_{B}$. This consideration leads to the conclusion that the Hilbert space is classified by the Cauchy horizon $H^{+}(B)$. 


\section{Applications of Strong Subadditivity}

Here we would like to apply the strong subadditivity relation (2.7) (2.9) to the entanglement entropy $S_{A}$ in conformal field theories in order to find constraints on the properties of $S_{A}$. Especially we are interested in $(2+1)$ and $(3+1)$ dimensional CFTs, though it is not difficult to extend our argument to higher dimensional theories.

\subsection{Entanglement Entropy in the Presence of Cusp}

Consider the entanglement entropy for a $(2+1)$ dimensional CFT. We assume that the spacetime is simply given by $R^{1,2}$. The subsystem $A$ (at a fixed time $t=0$ ) is geometrically described by a submanifold $A$ in the two dimensional space-like manifold $R^{2}$. When the boundary $\partial A$ is smooth (e.g. the case where $\partial A$ is a circle), we find the following behavior of the entropy

$$
S_{A}=\gamma \cdot \frac{|\partial A|}{a}+b
$$

where $a$ is the UV cutoff (lattice spacing), and $|\partial A|$ denotes the length of the boundary of $A$. Also $\gamma$ is a numerical constant. In general the leading divergent term is proportional to the area of the boundary $\partial A$, which is known as the area law [2]. The first term in (3.1) corresponds to this contribution. The constant $b$ does not depend on the cutoff $a$ and thus can be considered as a universal quantity. It depends on the explicit form of $\partial A$ in a conformal invariant way.

On the other hand, if the boundary $\partial A$ is singular and has a cusp, there exists a logarithmic correction as found quite recently in various systems [17] [19]. The cusp is specified by an angle defined such that $\Omega=\pi$ corresponds to a smooth line (see Fig.2). Therefore in this case the entropy takes the form

$$
S_{A}=\gamma \cdot \frac{|\partial A|}{a}+f(\Omega) \log a+b .
$$

Notice that the constant term $b$ depends on the cutoff $a$ in this case.

Now we would like to derive constraints on $f(\Omega)$ from the basic properties of entanglement entropy. First of all, by definition we find

$$
f(\pi)=0 .
$$



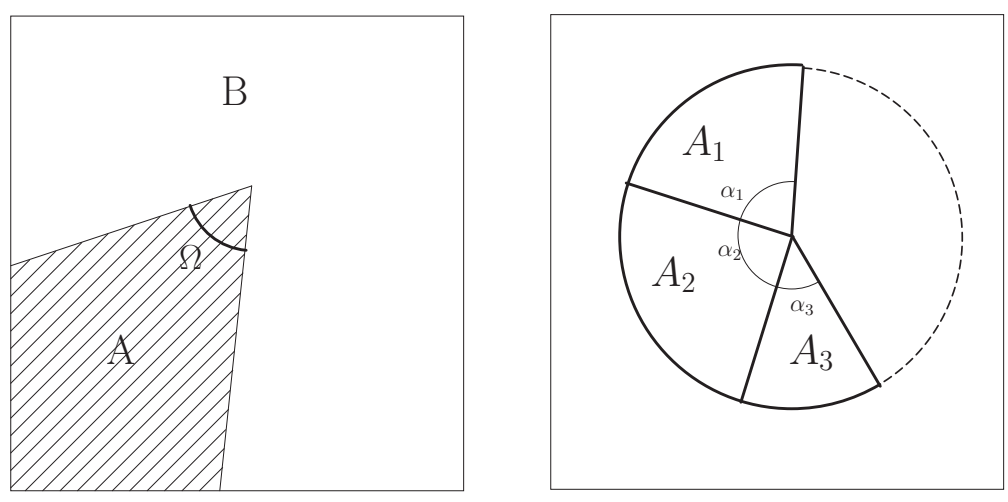

Fig. 2: The boundary with a cups (left). The setup to which the strong subadditivity is applied (right).

Also the equality $S_{A}=S_{B}$ (2.1) leads to

$$
f(\Omega)=f(2 \pi-\Omega)
$$

Next we would like to apply the strong subadditivity. Assume that the submanifolds $A_{1}, A_{2}$ and $A_{3}$ each have cusps with the angles $\alpha_{1}, \alpha_{2}$ and $\alpha_{3}$ such that one side of the cusp of $A_{1}$ (or $\left.A_{2}\right)$ is glued with that of $A_{2}$ (or $A_{3}$ ), respectively (thus we require $\sum_{i} \alpha_{i}<2 \pi$ ) as in Fig.2. Then the strong subadditivity leads to (note that leading divergences are all canceled out and that we finally take the limit $a \rightarrow 0$ )

$$
f\left(\alpha_{1}+\alpha_{2}+\alpha_{3}\right)-f\left(\alpha_{1}+\alpha_{2}\right) \geq f\left(\alpha_{2}+\alpha_{3}\right)-f\left(\alpha_{2}\right) .
$$

By taking the limit $\alpha_{3} \rightarrow+0$ we obtain

$$
f^{\prime}\left(\alpha_{1}+\alpha_{2}\right) \geq f^{\prime}\left(\alpha_{2}\right) \text {. }
$$

Finally the limit $\alpha_{1} \rightarrow+0$ leads to

$$
f^{\prime \prime}(\Omega) \geq 0
$$

Thus $f(\Omega)$ is a convex function 3 . Furthermore, the opposite is true, i.e. if $f$ is convex, then (3.5) does hold.

5 Even though the entropy is concave, the function $f$ is convex since the pre-factor $\log a$ is negative. 
It is also possible to combine the relation (3.4) with the strong subadditivity. Especially we take $\alpha_{1}=2 \pi-2 \alpha_{2}$. Then (3.5) can be reduced to $f\left(\alpha_{2}-\alpha_{3}\right) \geq f\left(\alpha_{2}+\alpha_{3}\right)$. Thus we find

$$
f^{\prime}(\Omega) \leq 0 \quad(\text { if } \quad \Omega \leq \pi)
$$

This leads to $f(\Omega) \geq 0$ due to $(3.3)$.

In conclusion, our results $(3.3),(3.7)$ and (3.8) can be summarized as follows

$$
f(\Omega) \geq 0, \quad f(\pi)=0, \quad f^{\prime}(\Omega) \leq 0, \quad f^{\prime \prime}(\Omega) \geq 0, \quad(0 \leq \Omega \leq \pi)
$$

For the values $\pi \leq \Omega \leq 2 \pi$ we can use the identity $f(\Omega)=f(2 \pi-\Omega)$.

\subsection{Entanglement Entropy for Annular Subsystem}

Next we turn to the case where the submanifold $A$ is an annulus such that its boundary $\partial A$ consists of two concentric rings with the radius $r_{1}$ and $r_{2}$ (assume $r_{1}<r_{2}$ ) as in Fig.3. We define its entanglement entropy by $S_{A}\left(r_{1}, r_{2}\right)$. As is clear from the discussions in the previous subsection, it takes the following general form

$$
S_{A}\left(r_{1}, r_{2}\right)=\gamma \cdot \frac{2 \pi\left(r_{1}+r_{2}\right)}{a}+b\left(\frac{r_{2}}{r_{1}}\right) .
$$

Notice that the universal finite term $b$ is a function of $r_{2} / r_{1}$ since it is dimensionless. There is no logarithmic term as opposed to the previous example because the boundary does not include any cusp singularity.
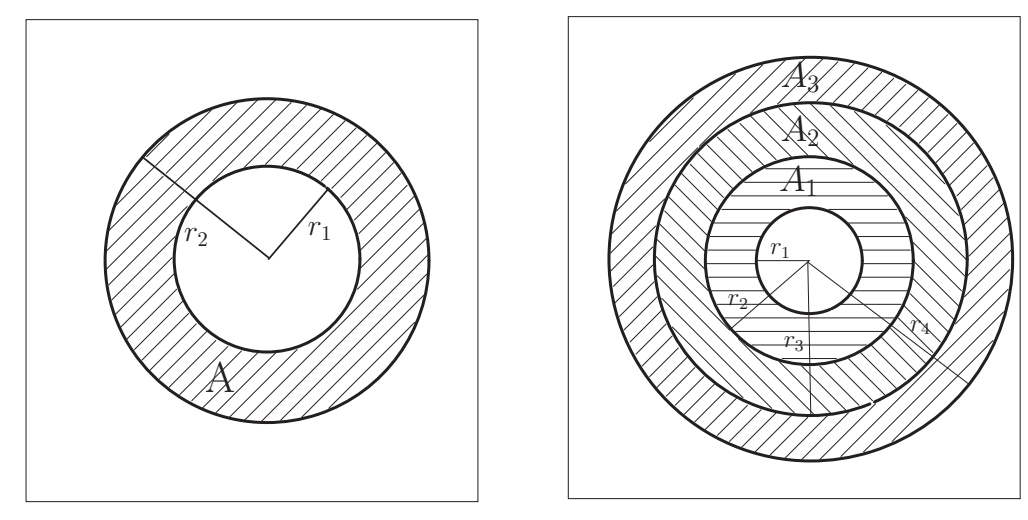

Fig. 3: The annulus boundary (left). The setup to which the strong subadditivity is applied (right). 
Now we would like to apply the strong subadditivity. Suppose three annular submanifolds $A_{1}, A_{2}$ and $A_{3}$ are placed successively such that the radii of their concentric rings are $\left(r_{1}, r_{2}\right),\left(r_{2}, r_{3}\right)$ and $\left(r_{3}, r_{4}\right)$, respectively $\left(r_{1}<r_{2}<r_{3}<r_{4}\right)$ as in Fig.3.

It is also useful to define $\rho_{i}=\log r_{i}$. Then it is straightforward to write down the inequality obtained from the strong subadditivity

$$
b\left(\rho_{4}-\rho_{1}\right)+b\left(\rho_{3}-\rho_{2}\right) \leq b\left(\rho_{3}-\rho_{1}\right)+b\left(\rho_{4}-\rho_{2}\right) .
$$

As a particular limit $\rho_{4}=\infty$ we find $b\left(\rho_{3}-\rho_{2}\right) \leq b\left(\rho_{3}-\rho_{1}\right)$. Thus we find

$$
b^{\prime}(\rho) \geq 0 .
$$

Also by taking the limits $\rho_{4} \rightarrow \rho_{3}$ and $\rho_{2} \rightarrow \rho_{1}$, we find

$$
b^{\prime \prime}(\rho) \leq 0 .
$$

Furthermore, the limit $\rho \rightarrow \infty$ is equivalent to the case where $A$ is a circular disk since the radius $r$ of the inner hole goes to zero. We write its value of $b$ as $b_{\text {disk }}$. In summary we have found the following properties of the function $b(\rho)\left(\rho=\log \frac{r^{\prime}}{r}>0\right)$

$$
b(\rho) \leq b(\infty)=b_{\text {disk }}, \quad b^{\prime}(\rho) \geq 0, \quad b^{\prime \prime}(\rho) \leq 0 .
$$

It is also intriguing to examine higher dimensional cases. The analogous boundary $\partial A$ can be obtained by replacing the two concentric rings with two such $d-1$ dimensional spheres. In particular, we concentrate on the most interesting case i.e. $d=3$ (4D CFT). The corresponding entanglement entropy takes the following form

$$
S_{A}=\gamma \cdot \frac{r_{1}^{2}+r_{2}^{2}}{a^{2}}-f \cdot \log \left(\frac{r_{1} r_{2}}{a^{2}}\right)+b\left(\frac{r_{2}}{r_{1}}\right) .
$$

The coefficient $f$ in front of the logarithmic term is universal and it is proportional to a linear combination of central charges in the 4D CFT [4] (see also [24] for earlier discussions). The general formula derived in [4] and the dual holographic computation via AdS/CFT discussed later show that $f=f\left(r_{2} / r_{1}\right)$ does not actually depend on $r_{1} / r_{2}$, i.e. just a constant. This is also clear from the observation that this term should be a local quantity since it originally depends on the cutoff $a$ and is divergent. Even though the function $b$ depends on the choice of the cutoff $a$, we can apply the strong subadditivity as before and obtain the properties (3.14) with $b(\infty)=b_{\text {disk }}$ now replaced by $b(\infty)=b_{\text {sphere. }}$. This is because both quadratic and logarithmic divergent term depend on the radii $r_{1}$ and $r_{2}$ only in the form $g\left(r_{1}\right)+g\left(r_{2}\right)$ and thus they are completely canceled out in the inequality of the strong subadditivity. 


\subsection{Entanglement Entropy for Straight Belt}

Before we move on to the holographic dual description, we would like to mention the simplest example of entanglement entropy. This is the case where the submanifold $A$ is given by the $d$ dimensional straight belt $A_{S}$ (refer also to [3] 团). It has the width $l$ in one direction and extends in other $d-1$ directions infinitely (its regularized length is denoted by $L$ ). In general, the entanglement entropy $S_{A}$ in $d+1 \geq 3$ dimensional CFT takes the following form

$$
S_{A}=\gamma \cdot \frac{L^{d-1}}{a^{d-1}}-\beta \cdot \frac{L^{d-1}}{l^{d-1}},
$$

where $\gamma$ and $\beta$ are positive constants. This form (3.16) has been obtained from the explicit computation in free field theories [25] and holographic results [3] [4]. The $d=1$ case is special and the entropy is known to be expressed as [5]

$$
S_{A}=\frac{c}{3} \log (l / a)
$$

where $c$ is the central charge.

Following discussions similar to the ones in the previous subsections, we can find that the strong subadditivity requires that the finite term $S_{\text {finite }}(l)$ of the entropy satisfies $\frac{d^{2}}{d l^{2}} S_{\text {finite }} \leq 0$. Indeed, this is clearly true for (3.16) and (3.17).

Moreover, we can obtain a stronger condition 6 from a relativistic consideration of the strong subadditivity as discovered in [16]. This requires

$$
\frac{d}{d l}\left(l \frac{d}{d l} S_{\text {finite }}\right) \leq 0 .
$$

Again this is satisfied by (3.16) and (3.17).

In two dimensional CFTs, (3.18) leads to the entropic c-theorem [15]. Define the entropic $c$-function $C$ by

$$
l \frac{d S_{A}}{d l}=C(l)
$$

in non-conformal field theories. $C(l)$ approaches $c / 3$ at a fixed point. Then (3.18) is equivalent to the c-theorem $C^{\prime}(l) \leq 0$ [15], regarding naturally $l$ as the length scale. It will be a very exciting future problem to extend this entropic proof of c-theorem to higher dimensions, though it does not seem to be straightforward.

6 The derivation of this stronger condition relies heavily on the simple structure of the submanifold $A$ and cannot be directly applied to other examples discussed in section 3.1 and 3.2. 


\section{Holographic Entanglement Entropy and Bousso Bound}

\subsection{Holographic Entanglement Entropy}

The holographic principle tells us that the true degree of freedom in a $d+2$ dimensional gravity theory is actually $d+1$ dimensional [26]. This idea is manifestly realized in string theory as the AdS/CFT correspondence [1]. In principle, we believe that we can compute any physical quantities in a $d+1$ dimensional CFT from the dual $d+2$ dimensional anti de-Sitter space $\left(A d S_{d+2}\right)$. Thus one direct way to obtain a gravitational interpretation of the entanglement entropy in a CFT is to apply the AdS/CFT correspondence.

In [3] [4], it is claimed that the entanglement entropy can be computed as follows

$$
S_{A}=\frac{A\left(\gamma_{A}\right)}{4 G_{N}^{(d+2)}}
$$

where $A(\Sigma)$ denotes the area of the surface $\Sigma$, and $G_{N}^{(d+2)}$ is the Newton constant in the $d+2$ dimensional anti de-Sitter space. The $d$ dimensional surface $\gamma_{A}$ is determined such that the minimal area surface whose boundary coincides with the boundary of submanifold A.

Intuitively, (4.1) can be understood by applying the Bekenstein-Hawking entropy formula to the surface $\gamma_{A}$ as if it is an event horizon as we expect that $\gamma_{A}$ represents the lost information hidden inside the region $B$ in gravity description. As checked in [3], the formula (4.1) is exactly true in $d=1$ examples. We can also find partial quantitative evidences in higher dimensional cases [4].

Moreover, as shown in [11] recently, we can derive (4.1) from the basic principle of AdS/CFT correspondence [12]. Its outline can be summarized very briefly as follows. Consider the partition function $Z_{C F T}$ of a CFT in the presence of the negative deficit angle $2 \pi-\delta<0$. It is the same as the $\operatorname{trace} \operatorname{tr}_{A} \rho_{A}^{n}$ by setting $n=\frac{\delta}{2 \pi}$. The locus of the deficit angle is localized on a codimension two surface which is identified with the boundary $\partial A$. Then the entanglement entropy in the CFT is equal to the derivative of partition function with respect to the deficit angle (refer to e.g. [4)

$$
S_{A}=-\left.\frac{\partial}{\partial n} \log \operatorname{tr}_{A} \rho_{A}^{n}\right|_{n=1}=-\left.2 \pi \frac{\partial}{\partial \delta} \log Z_{C F T}\right|_{\delta=2 \pi}
$$


On the other hand, the partition function in $\mathrm{CFT}_{d+1}$ can be obtained as that of the supergravity on the $\mathrm{AdS}_{d+2}$ space [12] via the AdS/CFT. At the tree level in the supergravity we thus find $Z_{C F T}=e^{-S_{\text {sugra }}}$, where $S_{\text {sugra }}$ is the tree level supergravity action. The deficit angle at the boundary $z=a$ is extended into the bulk of the AdS space and will form a $d$ dimensional surface $\gamma_{A}$. Then the Einstein-Hilbert term $\frac{1}{16 \pi G_{N}^{(d+2)}} \int d x^{d+2} \sqrt{G} R$ is evaluated as $\frac{A\left(\gamma_{A}\right)}{8 \pi G_{N}^{(d+2)}}(\delta-2 \pi)$ since the scalar curvature behaves as a delta function localized at the surface $\partial A$. Finally, we apply the ordinary variational principle we find that the surface $\gamma_{A}$ should be the minimal surface and it gives the largest contribution to the path-integral of the gravity partition function. In this way we recover (4.1) using (4.2).

\subsection{Relation to the Bousso Bound}

To apply the Bekenstein-Hawking formula to a surface which is actually not an event horizon, looks similar to the idea of the entropy bound [26] [13]. Motivated by this, in this subsection we would like to discuss the relation between entanglement entropy and covariant entropy bound, which is known as the Bousso bound [13].

The Bousso bound is the following claim. Consider a space-like codimension two manifold $\Sigma$ with the area $A(\Sigma)$. Its light-sheet $L(\Sigma)$ is defined to be a codimension one hypersurface bounded by $\Sigma$ and generated by one of the four null congruence orthogonal to $\Sigma$. We choose the light-sheet such that the expansion of light rays is always negative. Let $S_{L(\Sigma)}$ be the entropy on the light-sheet. Then the Bousso bound argues

$$
S_{L(\Sigma)} \leq \frac{A(\Sigma)}{4 G_{N}}
$$

We would like to apply this bound to the spacetime $A d S_{d+2}$.

Now we go back to the entanglement entropy in CFTs. Physically the entanglement entropy $S_{A}$ measures the loss of information when we smear out the region $B$. As we have explained, $S_{A}$ is specified by the Cauchy horizon $H^{+}(B)$. Thus its bulk description should be such that the light-sheet, where the amount of information (or entropy) is measured, ends on $H^{+}(B)$ at the boundary. Since $L(\Sigma)$ is defined such that $L(\Sigma) \perp \Sigma$, the spacelike manifold $\Sigma$ is orthogonal to the boundary when they meet. This is always satisfied if we 


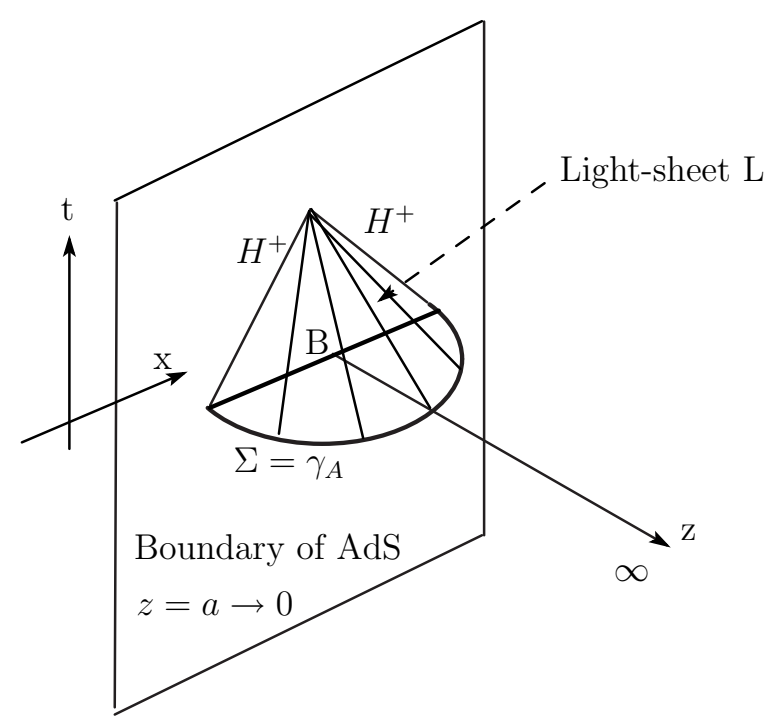

Fig. 4: Relation to Bousso bound. We write the light-sheet in the Poincare coordinate $d s^{2}=z^{-2}\left(-d t^{2}+d x^{2}+d z^{2}\right)$ of $A d S_{3}$. Notice the form of lightcone is the same as that in the flat spacetime. The light-sheet is given by a half cone $L$ and the minimal surface $\gamma_{A}$ is a half circle.

choose $\Sigma$ the minimal surface $\gamma_{A}$. Notice that, however, we can choose other surface with this property. In this way we find the setup in Fig.4.

Now we apply the Bousso bound to this system. The entropy on the light-sheet is bounded by the area of $\Sigma$. If $\Sigma$ is chosen to be the minimal surface $\gamma_{A}$, we find $S_{L\left(\gamma_{A}\right)} \leq$ $\frac{A\left(\gamma_{A}\right)}{4 G_{N}}$ from (4.3). Following the general idea of holography we would like to claim that the entanglement entropy is the same as the entropy on the light-sheet $L\left(\gamma_{A}\right)$. Then the relation (4.1) claims that the entanglement entropy $S_{A}$ saturates the Bousso bound (4.3)

$$
S_{A}=S_{L\left(\gamma_{A}\right)}=\frac{A\left(\gamma_{A}\right)}{4 G_{N}}
$$

All of these claims can be shown to be exactly true in the $A d S_{3} / C F T_{2}$ case as the entanglement entropies in 2D CFTs are analytically computable [3]. Also notice that even if we do not choose $\gamma_{A}$ as $\Sigma$, the previous condition that $\Sigma$ should be orthogonal to the boundary is enough to prove that the leading divergent term in $\frac{A(\Sigma)}{4 G_{N}}$ is the same as the leading (area law) term 1 in the holographic entanglement entropy (4.1).

7 It is clear that these arguments also hold for asymptotically AdS spaces. The dual theories are quantum field theories with UV fixed points. One of the examples which do not satisfy this 
We also need to explain why the bound is saturated. One explanation is clearly to remember the proof found in [11] and to directly compute the entropy following the basic principle of AdS/CFT [12]. Even if we do not apply the AdS/CFT, we can at least tell that this is strongly suggested. This is because we are choosing the minimal surfaces among other candidates of $\Sigma$. If we employ any one of the others, we will have a weaker bound since $A\left(\gamma_{A}\right)<A(\Sigma)$ for all $\Sigma$ s other than $\gamma_{A}$. Thus the minimal surface offers the strictest bound in (4.3) and thus there is an opportunity to saturate the bound.

These arguments clarifies the following important question. In which region of the $A d S$ space is encoded the information in the dual CFT? Our example depicted in Fig.4 manifestly shows that the information in the $B$ region, which is equal to the one in its causal development $D^{+}(B)$, is dual to the inside of the light-sheet $L$.

\section{Holographic Evidences for Strong Subadditivity}

In this section we would like to discuss the main issue of this paper. This is to examine if the strong subadditivity is satisfied in the holographic dual computations. For this purpose, we will study several particular examples of entanglement entropy in $2+1$ and $3+1$ dimensional CFTs. In the dual $A d S_{d+2}$ spacetime, we always employ the Poincare coordinate

$$
d s^{2}=R^{2} \frac{d z^{2}-d x_{0}^{2}+\sum_{i=1}^{d} d x_{i}^{2}}{z^{2}}
$$

The radial coordinate $z$ represents the length scale and we put the UV cutoff at $z=a$. To compute the entanglement entropy, we choose a particular time slice e.g. $x_{0}=0$ and then try to find the minimal surface $\gamma_{A}$. We require that the boundary of $\gamma_{A}$ at $z=a$ coincides with the boundary of the submanifold $A$ (or equally its complement $B$ ) which defines the

criterion is the $4 \mathrm{D}$ cascading theory [27]. In this model, The radius of the five dimensional Einstein manifold $R$ depends on the energy scale or equally the radial coordinate $r$ as $R \sim(\log r)^{\frac{1}{4}}$ in the UV region. This corresponds to the fact that the rank of gauge groups increases at higher energy in the cascading gauge theory. The calculation of minimal surface areas can be done as before and in the end we find that the leading divergence is proportional to $-\frac{|\partial A|}{a^{2}} \cdot(\log a)^{2}$. Thus in this case, the well-known area law of the leading divergence $\sim \frac{|\partial A|}{a^{d-1}}$ (see also (3.16)) is modified by the logarithmic factor. 
entanglement entropy $S_{A}$ as explained in section 4. Finally we apply the formula (4.1) to obtain the entropy $S_{A}$. Notice that this is generally divergent in the limit $a \rightarrow 0$ and the

leading divergence takes the form $\sim \frac{|\partial A|}{a^{d-1}}$, which agrees with the known area law of the entanglement entropy.

The results found for $(2+1)$ dimensional CFTs via holographic computations can be equally applied to the calculation of Wilson loops in $(3+1)$ dimensional CFTs which are defined by strongly coupled gauge theories. Therefore we can claim that the strong subadditivity relation is also true for the Wilson loop correlation functions. This issue will be discussed briefly in the section 6 .

\subsection{Cusp Case}

First we would like to discuss the $d=2$ example (i.e. $(2+1)$ dim. CFT) where the subsystem $A$ has a cusp singularity. This is the same setup as the one discussed in section 3.1. The boundary $\partial A$ is a line with a cusp at a point as in Fig.2. Its angle is defined to be $\Omega$. In the polar coordinate $(r, \phi)$ of the $\left(x_{1}, x_{2}\right)$-plane, it is just described by

$$
\{(r, \phi) \mid 0 \leq r<\infty, \phi=0\} \cup\{(r, \phi) \mid 0 \leq r<\infty, \phi=\Omega\}
$$

Next we would like to find the corresponding minimal surface $\gamma_{A}$ in $A d S_{d+2}$. Fortunately, this setup is essentially the same as that appears in the Wilson loop calculation [20] done in 28] and thus we can employ the results there.

Using the conformal symmetry $z \rightarrow \lambda z, x_{\mu} \rightarrow \lambda x_{\mu}$, we can assume that the surface $\gamma_{A}$ is described 28 by

$$
z(r, \phi)=\frac{r}{g(\phi)}
$$

The area of $\gamma_{A}$ is given by

$$
\left|\gamma_{A}\right|=R^{2} \int \frac{d r}{r} \int d \phi \sqrt{\left(g^{\prime}\right)^{2}+g^{2}+g^{4}}
$$

Thinking $\phi$ as a time, we find the Hamiltonian

$$
H=\frac{\partial L}{\partial g^{\prime}} g^{\prime}-L=-\frac{g^{2}+g^{4}}{\sqrt{\left(g^{\prime}\right)^{2}+g^{2}+g^{4}}}=\text { const. }\left(\equiv-g_{0}^{2}-g_{0}^{4}\right) \text {. }
$$


Then we can reduce the minimal surface equation to the following differential equation

$$
\frac{d g}{d \phi}=g \sqrt{\left(1+g^{2}\right)\left(\frac{g^{2}+g^{4}}{g_{0}^{2}+g_{0}^{4}}-1\right)} .
$$

Since $g=g_{0}$ is the turning point, we have to require

$$
\begin{aligned}
\frac{\Omega}{2} & =\int_{g_{0}}^{\infty} \frac{d g}{g \sqrt{\left(1+g^{2}\right)\left(\frac{g^{2}+g^{4}}{g_{0}^{2}+g_{0}^{4}}-1\right)}} \\
& =g_{0} \sqrt{1+g_{0}^{2}} \int_{0}^{\infty} \frac{d z}{\left(z^{2}+g_{0}^{2}\right) \sqrt{\left(z^{2}+g_{0}^{2}+1\right)\left(z^{2}+2 g_{0}^{2}+1\right)}}
\end{aligned}
$$

where we set $g^{2}=z^{2}+g_{0}^{2}$.

In the end the minimal area can be found as

$$
\begin{aligned}
\left|\gamma_{A}\right| & =2 R^{2} \int_{g_{0} a}^{\infty} \frac{d r}{r} \int_{g_{0}}^{r / a} d g \frac{g \sqrt{1+g^{2}}}{\sqrt{\left(g^{2}-g_{0}^{2}\right)\left(g^{2}+g_{0}^{2}+1\right)}} \\
& =2 R^{2} \int_{g_{0} a}^{\infty} \frac{d r}{r} \int_{0}^{r / a} d z \sqrt{\frac{z^{2}+g_{0}^{2}+1}{z^{2}+2 g_{0}^{2}+1}}
\end{aligned}
$$

where we noticed that the upper bound of the $g$ integral is $r / a$ from (5.3). The integration of $g$ diverges as $\frac{r}{a}-f(\Omega)$ when the cutoff $a$ is set to zero. The finite part is explicitly given by

$$
f(\Omega)=\int_{0}^{\infty} d z\left[1-\sqrt{\frac{z^{2}+g_{0}^{2}+1}{z^{2}+2 g_{0}^{2}+1}}\right],
$$

where the relation between $\Omega$ and $g_{0}$ is determined from (5.7). Then the total area can be found as (the upper bound of $r$ is defined to be $L$ for a regularization)

$$
\frac{\left|\gamma_{A}\right|}{R^{2}}=\frac{2 L}{a}-2 f(\Omega) \log \frac{L}{a}+(\text { finite terms })
$$

Thus the entanglement entropy is now computed as (we omit finite constant terms)

$$
S_{A}=\frac{R^{2}}{4 G_{N}^{(4)}}\left(\frac{2 L}{a}-2 f(\Omega) \log \frac{L}{a}\right) .
$$

Note that the function $f(\Omega)$ is the same as the one in (3.2) up to the factor $\frac{R^{2}}{2 G_{N}^{(4)}}$. 


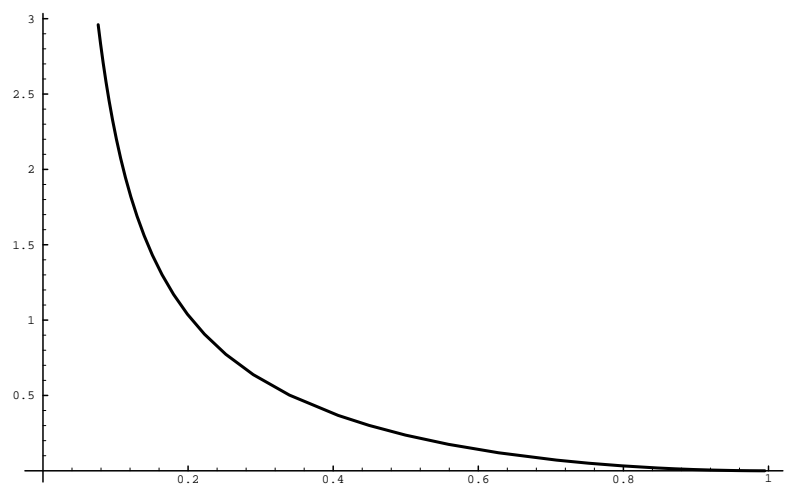

Fig. 5: The function $f(\Omega)$ in $(2+1) \mathrm{D}$ CFT; the horizontal and vertical coordinate are given by $\Omega / \pi$ and $f(\Omega)$, respectively. $f(\Omega)$ becomes vanishing at $\Omega=\pi$.

The strong subadditivity tells us that the function $f(\Omega)$ is a convex function as we have seen in section 3.1. Indeed we can check this property from the explicit form of $f(\Omega)$ shown in Fig.5.

It is also possible to find the following analytic form of $f(\Omega)$ when $\Omega$ is very small

$$
f(\Omega) \simeq \frac{\Gamma\left(\frac{3}{4}\right)^{4}}{\pi} \cdot \frac{1}{\Omega}
$$

This behavior $f(\Omega) \propto \Omega^{-1}$ for a small angle is the same as the one computed in the free scalar field theory ${ }^{\mathrm{B}}$ [17].

Before we jump to the next example, it may be helpful to apply our formula (5.11) to a specific background. Consider the $A d S_{4} \times S^{7}$ realized as the near horizon limit of $N$ M2-branes in M-theory . The corresponding CFT is defined by the strong coupling limit of the $(2+1)$ dimensional maximally supersymmetric gauge theory. The entropy is explicitly given by

$$
S_{A}=\frac{N^{\frac{3}{2}}}{3 \sqrt{2}}\left(\frac{2 L}{a}-2 f(\Omega) \log \frac{L}{a}\right) .
$$

8 In the free field theory, the coefficient $\beta$ in front of $\Omega^{-1}$ in the logarithmic term of $S_{A}$ $\left(\beta=\frac{R^{2}}{2 G_{N}^{(4)}} \cdot \frac{\Gamma\left(\frac{3}{4}\right)^{4}}{\pi}\right.$ in our case of $\left.(5.12)\right)$ is equal to $\frac{1}{\pi} \int_{0}^{\infty} d t c(t)$, where $c(t)$ is the entropic cfunction [17]. Also in the same paper [17] it is argued and confirmed in the free field theoretic computations that this quantity $\beta$ is equal to the coefficient $\beta$ of the entropy in the straight line case (see (3.16) ). We can check that this relation is indeed true in our case exactly. We are very grateful to H. Casini for explaining this relation to us. 


\subsection{Annulus Case}

Next we examine the case where the subsystem $A$ is given by the annulus whose boundary $\partial A$ consists of two concentric rings with the radius $r_{1}$ and $r_{2}$ (assume $r_{1}<r_{2}$ ). We again use the polar coordinate for the $\left(x_{1}, x_{2}\right)$-plane. We can also consider higher dimensional cases where the concentric rings are replaced by concentric $d-1$ dimensional spheres. These are the same setups as the ones discussed in section 3.2. Below we will mainly be interested in the $d=2$ and $d=3$ case (i.e. $(2+1)$ and $(3+1)$ dimensional CFTs).

The minimal surface is the $d$ dimensional 'half torus' defined by the half circle $z=$ $z(r)$ times the sphere $S^{d-1}$ s.t. $z\left(r_{1}\right)=z\left(r_{2}\right)=a \rightarrow 0$. The function $z(r)$ is found by minimizing the area functional

$$
\left|\gamma_{A}\right|=R^{d} \cdot \operatorname{Vol}\left(\mathrm{S}^{d-1}\right) \cdot \int_{r_{1}}^{r_{2}} d r r^{d-1} \frac{\sqrt{1+\left(\frac{d z}{d r}\right)^{2}}}{z^{d}}
$$

We can find the following equation of motion

$$
r z z^{\prime \prime}+(d-1) z\left(z^{\prime}\right)^{3}+(d-1) z z^{\prime}+d r\left(z^{\prime}\right)^{2}+d r=0 .
$$

Its simplest solution is the $d$ dimensional sphere $z^{2}+r^{2}=$ const. Clearly we have to deal with more complicated solutions to find the desired configurations. Thus one way to analyze this problem is to directly resort a numerical analysis as explained below.

First let us concentrate on the $d=2$ case. The numerical investigation shows that there is an upper limit on the allowed value of $r_{2} / r_{1}$. This means that if $r_{2} / r_{1}$ is large enough, then there is no solution to (5.14). In this case the minimal area surface should be regarded as the two disconnected spheres with the radii $r_{1}$ and $r_{2}$. This critical value can be estimated as $\left(r_{2} / r_{1}\right)_{*} \simeq 2.725$. This kind of phase transition has already been known in the context of Wilson loop computations and is called Gross-Ooguri transition 29] 30]. We solved (5.15) numerically and calculated the finite part of the integral (setting $\rho=\log \left(r_{2} / r_{1}\right)$ )

$$
\tilde{b}(\rho)=\left[\int_{r_{1}}^{r_{2}} d r r \frac{\sqrt{1+\left(\frac{d z}{d r}\right)^{2}}}{z^{2}}\right]-\frac{r_{1}+r_{2}}{a} .
$$


The result is plotted in Fig.6. The total entanglement entropy is expressed as

$$
S_{A}=\frac{\left|\gamma_{A}\right|}{4 G_{N}^{(4)}}=\frac{2 \pi R^{2}}{4 G_{N}^{(4)}}\left(\frac{r_{1}+r_{2}}{a}+\tilde{b}(\rho)\right) .
$$

The entanglement entropy 9 in the specific example of $A d S_{4} \times S^{7}$ is obtained by substituting $\frac{R^{2}}{4 G_{N}^{(4)}}=\frac{N^{\frac{3}{2}}}{3 \sqrt{2}}$.

Now we can see that there are two branches of solutions when $r_{2} / r_{1}<\left(r_{2} / r_{1}\right)_{*}$ as we showed in Fig.6. One of them is uninteresting since its area is larger than the trivial one (i.e. two disconnected spheres $\tilde{b}_{\text {sphere }}=-2$ ). The second one is physically relevant as long as $\tilde{b}<-2$ (i.e. $\rho<0.88$ ). Above that point, the trivial one becomes more stable and thus we have $\tilde{b}(\rho)=-2$.

In summary, the physically relevant minimal surface is found as follows. When $\tilde{b}<\tilde{b}_{\text {sphere }}$, it is given by one of the two non-trivial connected solutions to (5.15) with the smaller area. At $\tilde{b}=\tilde{b}_{\text {sphere }}$, a transition takes place. Then it becomes the disconnected sphere solution when $\tilde{b}>\tilde{b}_{\text {sphere }}$. Therefore we can check from Fig. 6 that the finite part $b(\rho)=\frac{2 \pi R^{2}}{4 G_{N}^{(4)}} \tilde{b}(\rho)$ of the entanglement entropy is a concave function of $\rho$ (i.e. $\left.b^{\prime \prime} \leq 0\right)$ and this agrees with the strong subadditivity discussed in section 3.2. It may also be intriguing to note that the first irrelevant solution does not satisfy the concave condition. For that solution, the value of $\tilde{b}$ approaches $\tilde{b}_{\text {sphere }}=-2$ in the $\rho \rightarrow 0$ limit.

Now we turn to the $d=3$ case. The entropy takes the following form

$$
S_{A}=\left(\frac{R^{3}}{4 G_{N}^{(5)}}\right) \cdot 4 \pi\left[\frac{r^{2}+r^{\prime 2}}{2 a^{2}}-\frac{1}{2} \log \left(\frac{r r^{\prime}}{a^{2}}\right)+\tilde{b}_{3}\left(\frac{r^{\prime}}{r}\right)\right] .
$$

In the specific case of $A d S_{5} \times S^{5}$ dual to the $\mathcal{N}=4 S U(N)$ super Yang-Mills, the entropy is given by substituting $\frac{R^{3}}{4 G_{N}^{(5)}}=\frac{N^{2}}{2 \pi}$ in (5.18). The coefficient of leading divergence and logarithmic divergence in (5.18) can be fixed by noting that when $z$ is small, the solution to (5.15) is well approximated by the simple sphere solutions 10 .

9 We can also apply the above result for $d=2$ to the Wilson loop computation. This leads to the following correlation function of two concentric Wilson loop operators in the strongly coupled large $N \mathcal{N}=4$ super Yang-Mills theory $\left\langle W(r) W\left(r^{\prime}\right)\right\rangle^{\text {connected }}=e^{-\sqrt{2 g_{Y M}^{2} N \cdot \tilde{b}(\rho)}}$.

10 This fact can be checked by looking at the asymptotic behavior at $z=0$ of the function $r=r(z)$, which satisfies the differential equation $z r r^{\prime \prime}-(d-1)\left(r^{\prime}\right)^{2} z-(d-1) z-d r r^{\prime}-d r\left(r^{\prime}\right)^{3}=0$ equivalent to (5.15). 


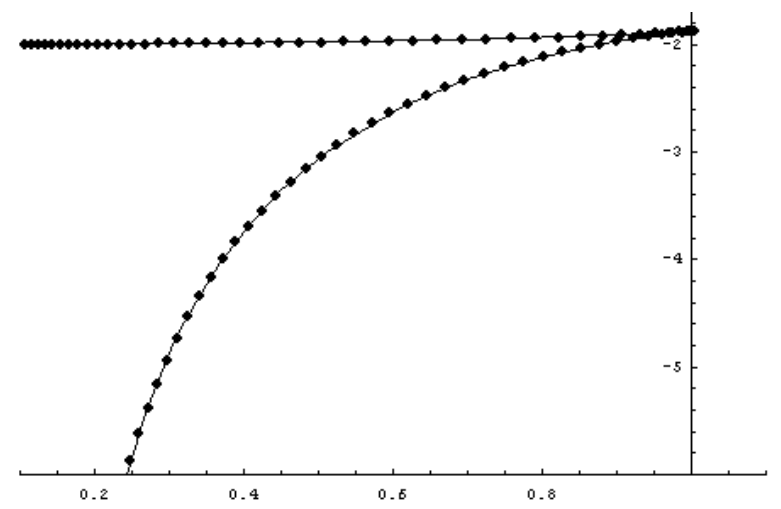

Fig. 6: The function $\tilde{b}(\rho)$ computed from the minimal surfaces in $A d S_{4}$ $(d=2)$. The horizontal and vertical coordinate are $\rho$ and $\tilde{b}$, respectively. The dotted points are obtained from the direct numerical solutions to (5.15), while the solid curves are from the analytical method discussed in (5.19). Both of them agree with each other very well. There are two solutions and the lower one is physically relevant as it has a smaller area.

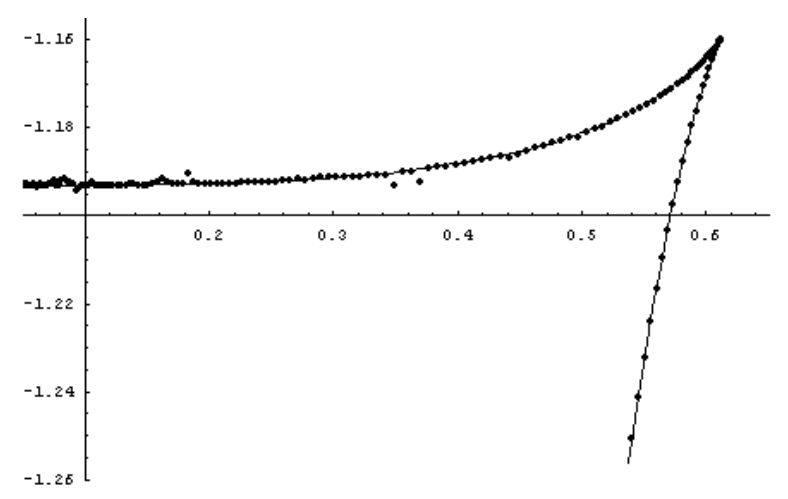

Fig. 7: The function $\tilde{b}_{3}(\rho)$ computed from the minimal surfaces in $A d S_{5}$ $(d=3)$. The horizontal and vertical coordinate are $\rho$ and $\tilde{b}$, respectively. The dotted points are obtained from the direct numerical solutions to (5.15), while the solid curves are from the analytical method discussed in (5.21). Both of them agree with each other very well. There are two solutions and the lower one is physically relevant as it has a smaller area.

We again solved (5.15) numerically and computed the finite term $\tilde{b}_{3}$. This is plotted in Fig.7. The properties of the solutions to (5.15) at $d=3$ are similar to the previous one at $d=2$. Again two different solutions exist when $r_{2} / r_{1}<\left(r_{2} / r_{1}\right)_{*}=1.844$. Above this value there is no connected solution. The value of $\tilde{b}_{3}$ for one of the two solutions is always greater than that of two disconnected spheres $\tilde{b}_{3 s p h e r e}=-1 / 2-\log 2 \simeq-1.193$ and it approaches this value $\tilde{b}_{3 s p h e r e}$ in the $\rho \rightarrow 0$ limit. Thus this solution is physically irrelevant. 
In summary, the physically relevant minimal surface is found as follows. When $\tilde{b}_{3}<\tilde{b}_{3 s p h e r e}$, it is given by one of the two non-trivial connected solutions to (5.15) with the smaller area. At $\tilde{b}_{3}=\tilde{b}_{3 s p h e r e}$, a transition takes place. Then it becomes the disconnected sphere solution when $\tilde{b}_{3}>\tilde{b}_{3 \text { sphere }}$. Thus again we can confirm that this function $\tilde{b}_{3}(\rho)$ is concave, agreeing with the strong subadditivity.

Finally we would like to briefly mention a semi-analytical approach to this problem. Let us artificially add an extra coordinate $x^{d+1}$ in (5.1) (i.e. $A d S_{d+3}$ ) and consider the $d$ dimensional minimal surface whose boundary is given by two concentric rings (or spheres). We first assume that they are separated from each other by $\Delta x^{d+1}$ in the new $x^{d+1}$ direction. When $d=2$, this example has been studied in [30] [31] in order to compute the Wilson loops. In this setup, we can find first integrals and reduce the problem to the analysis of first order differential equations 1 [30] [31] if we treat all quantities as functions of $x^{d+1}$.

Further, we take the zero separation limit $\Delta x^{d+1} \rightarrow 0$. Then we go back to the original setup discussed in the above to compute the entanglement entropy (i.e. (5.15)). However, we have to be careful since the radii of the rings become zero in this limit. If we neglect this point, we can find the integral expression of the area $\left|\gamma_{A}\right|$. These have already been expressed in Fig.6 and Fig.7 as a solid curve. Indeed, we can see that they nicely agree with the results obtained by solving (5.15) numerically. Thus we can find that this method offers us the exact computations of $\tilde{b}$ and $\tilde{b}_{3}$.

Their explicit forms are summarized as follows. In the $d=2$ case, we first define the parameter $s$ in terms of $\rho\left(\equiv \log \left(r_{2} / r_{1}\right)\right)$ as follows

$$
\rho=s \int_{0}^{\sin ^{2} \theta_{0}} d y \frac{y^{1 / 2}}{\sqrt{(1-y)\left(1-y-s^{2} y^{2}\right)}},
$$

where $\theta_{0}$ is the solution to $s^{2} \sin ^{4} \theta_{0}=\cos ^{2} \theta_{0}$. Then the area becomes

$$
\frac{\left|\gamma_{A}\right|_{d=2}}{2 \pi R^{2}}=\int_{a / r_{1}}^{\theta_{0}} d \theta \frac{\cos \theta^{2}}{\sin \theta^{2} \sqrt{\cos ^{2} \theta-s^{2} \sin ^{4} \theta}}+\int_{a / r_{2}}^{\theta_{0}} d \theta \frac{\cos \theta^{2}}{\sin \theta^{2} \sqrt{\cos ^{2} \theta-s^{2} \sin ^{4} \theta}} .
$$

11 After we submitted this paper, we noticed that in the recent paper [32], the explicit solution at $\Delta x^{d+1}=0$ was obtained in the context of Wilson loop. We are grateful to N. Drukker for letting us know the paper. 
In the $d=3$ case, we define

$$
\rho=s \int_{0}^{\sin ^{2} \theta_{0}} d y \frac{y}{\sqrt{(1-y)\left((1-y)^{2}-s^{2} y^{3}\right)}}
$$

where $\theta_{0}$ is the solution to $s^{2} \sin ^{6} \theta_{0}=\cos \theta_{0}^{4}$. Finally the area becomes

$$
\frac{\left|\gamma_{A}\right|_{d=3}}{4 \pi R^{3}}=\int_{a / r_{1}}^{\theta_{0}} d \theta \frac{\cos \theta^{4}}{\sin \theta^{3} \sqrt{\cos ^{4} \theta-s^{2} \sin ^{6} \theta}}+\int_{a / r_{2}}^{\theta_{0}} d \theta \frac{\cos \theta^{4}}{\sin \theta^{3} \sqrt{\cos ^{4} \theta-s^{2} \sin ^{6} \theta}}
$$

The functions $\tilde{b}(\rho)$ and $\tilde{b}_{3}(\rho)$ can be obtained from (5.20) and (5.22) by subtracting the divergences.

\section{Conclusions and Discussions}

In this paper, we explored further evidences for the recent proposal of holographic computations of entanglement entropy. Especially we looked at an important property of the entanglement entropy, known as the strong subadditivity.

First we showed that this requires various terms in the entanglement entropy should be concave functions with respect to the geometric parameters such as the cusp angle or the ratio of radii of an annulus. Secondly we computed the entanglement entropy from the dual gravity side in several explicit examples and found that the strong subadditivity is satisfied in all of our examples. This offers us a highly non-trivial check of our proposal. It will be obviously an important future problem to derive the required concavity of the minimal surface area in AdS spaces from a more systematic method, which probably needs the use of a positive energy condition and of the Einstein equation on AdS spaces.

Our results in $(2+1) \mathrm{D}$ CFT can directly be applied to the Wilson loop computations in $4 \mathrm{D}$ gauge theories via the AdS/CFT correspondence. Thus we are led to the conjecture that the strong subadditivity relation (2.9) is also true for the (locally supersymmetric) Wilson loops $\langle W(C)\rangle$ by replacing $S_{A}$ with $-\log \langle W(\partial A)\rangle$ at least in strongly coupled gauge theories. Indeed, the concavity of the quark-antiquark potential has been already shown in [33], which corresponds to the particular case of rectangular Wilson loops. This property has been successfully tested in the AdS/CFT correspondence in [34] 355] for some specific examples. This issue clearly deserves further investigations. 
In addition, we discussed the relation between the holographic entanglement entropy and the covariant entropy bound known as the Bousso bound. We noted that the holographic entanglement entropy formula (4.1) implies that the entropy bound is saturated. This leads to another natural explanation of the proposal. At the same time, this makes clear the region in AdS space where are encoded the information included in a specified submanifold of the boundary CFT.

\section{Acknowledgments}

We are extremely grateful to $\mathrm{H}$. Casini and S. Ryu for the careful reading of the manuscript and for valuable discussions. We thanks A. Shirasaka and S. Yamato for useful discussions, T. Azeyanagi for careful reading and useful comments, and T. Muranushi for technical supports on numerical computations. We would also like to thank N. Drukker

for letting us know relevant and useful papers. The research of $\mathrm{TH}$ was supported in part by JSPS Research Fellowships for Young Scientists. 


\section{References}

[1] J. M. Maldacena, "The large N limit of superconformal field theories and supergravity," Adv. Theor. Math. Phys. 2, 231 (1998) [Int. J. Theor. Phys. 38, 1113 (1999)] arXiv:hep-th/9711200.

[2] L. Bombelli, R. K. Koul, J. H. Lee and R. D. Sorkin, "A Quantum Source of Entropy for Black Holes," Phys. Rev. D 34, 373 (1986); M. Srednicki, "Entropy and area," Phys. Rev. Lett. 71, 666 (1993) arXiv:hep-th/9303048.

[3] S. Ryu and T. Takayanagi, "Holographic derivation of entanglement entropy from AdS/CFT," Phys. Rev. Lett. 96, 181602 (2006) arXiv:hep-th/0603001.

[4] S. Ryu and T. Takayanagi, "Aspects of holographic entanglement entropy," arXiv:hepth/0605073.

[5] C. Holzhey, F. Larsen and F. Wilczek, "Geometric and renormalized entropy in conformal field theory," Nucl. Phys. B 424, 443 (1994) arXiv:hep-th/9403108; P. Calabrese and J. L. Cardy, "Entanglement entropy and quantum field theory," J. Stat. Mech. 0406, P002 (2004) arXiv:hep-th/0405152.

[6] R. Emparan, "Black hole entropy as entanglement entropy: A holographic derivation," JHEP 0606, 012 (2006) [arXiv:hep-th/0603081.

[7] S. N. Solodukhin, "Entanglement entropy of black holes and AdS/CFT correspondence," arXiv:hep-th/0606205.

[8] Y. Iwashita, T. Kobayashi, T. Shiromizu and H. Yoshino, "Holographic entanglement entropy of de Sitter braneworld," arXiv:hep-th/0606027.

[9] S. Hawking, J. M. Maldacena and A. Strominger, "DeSitter entropy, quantum entanglement and AdS/CFT," JHEP 0105, 001 (2001) arXiv:hep-th/0002145.

[10] J. M. Maldacena, "Eternal black holes in Anti-de-Sitter," JHEP 0304, 021 (2003) arXiv:hep-th/0106112.

[11] D. V. Fursaev, "Proof of the holographic formula for entanglement entropy," arXiv:hep-th/0606184.

[12] S. S. Gubser, I. R. Klebanov and A. M. Polyakov, "Gauge theory correlators from non-critical string theory," Phys. Lett. B 428, 105 (1998) arXiv:hep-th/9802109; E. Witten, "Anti-de Sitter space and holography," Adv. Theor. Math. Phys. 2, 253 (1998) arXiv:hep-th/9802150.

[13] R. Bousso, "A Covariant Entropy Conjecture," JHEP 9907, 004 (1999) arXiv:hepth/9905177]; "Holography in general space-times," JHEP 9906, 028 (1999) arXiv:hepth/9906022].

[14] E. H. Lieb and M. B. Ruskai, "Proof of the strong subadditivity of quantummechanical entropy," J. Math. Phys. 14, 1938 (1973).

[15] H. Casini and M. Huerta, "A finite entanglement entropy and the c-theorem," Phys. Lett. B 600, 142 (2004) arXiv:hep-th/0405111. 
[16] H. Casini, "Geometric entropy, area, and strong subadditivity," Class. Quant. Grav. 21, 2351 (2004) arXiv:hep-th/0312238.

[17] H. Casini and M. Huerta, "Universal terms for the entanglement entropy in $2+1$ dimensions," arXiv:hep-th/0606256.

[18] D. V. Fursaev, "Entanglement entropy in critical phenomena and analogue models of quantum gravity," Phys. Rev. D 73, 124025 (2006) arXiv:hep-th/0602134.

[19] E. Fradkin and J. E. Moore, "Entanglement entropy of 2D conformal quantum critical points: hearing the shape of a quantum drum," arXiv:cond-mat/0605683.

[20] J. M. Maldacena, "Wilson loops in large N field theories," Phys. Rev. Lett. 80, 4859 (1998) arXiv:hep-th/9803002; S. J. Rey and J. T. Yee, "Macroscopic strings as heavy quarks in large N gauge theory and anti-de Sitter supergravity," Eur. Phys. J. C 22, 379 (2001) arXiv:hep-th/9803001.

[21] H. Araki and E. H. Lieb, "Entropy inequalities," Commun. Math. Phys. 18, 160 (1970).

[22] See for example, M. Nielsen, and I. L. Chuang, "Quantum Computation and Quantum Information", Cambridge university press, (2000).

[23] J. Aczel, B. Forte and C. T. Ng, "Why the Shannon and Hartley entropies are natural," Adv. Appl. Prob. bf 6 (1974), 131; W. Ochs, "A new axiomatic characterization of the von Neumann entropy," Rep. Math. Phys. 8 (1975), 109; A. Wehrl, "General properties of entropy," Rev. Mod. Phys. 50, 1978, 221.

[24] S. N. Solodukhin, "The Conical singularity and quantum corrections to entropy of black hole," Phys. Rev. D 51, 609 (1995) arXiv:hep-th/9407001; "On 'Nongeometric' contribution to the entropy of black hole due to quantum corrections," Phys. Rev. D 51, 618 (1995) arXiv:hep-th/9408068; "One loop renormalization of black hole entropy due to nonminimally coupled matter," Phys. Rev. D 52, 7046 (1995) arXiv:hep-th/9504022.

[25] H. Casini, C. D. Fosco and M. Huerta, "Entanglement and alpha entropies for a massive Dirac field in two dimensions," J. Stat. Mech. 0507, P007 (2005) arXiv:condmat/0505563; H. Casini and M. Huerta, "Entanglement and alpha entropies for a massive scalar field in two dimensions," J. Stat. Mech. 0512, P012 (2005) arXiv:condmat/0511014.

[26] L. Susskind, "The World As A Hologram," J. Math. Phys. 36, 6377 (1995) arXiv:hepth/9409089].

[27] I. R. Klebanov and A. A. Tseytlin, "Gravity duals of supersymmetric SU(N) x SU(N+M) gauge theories," Nucl. Phys. B 578, 123 (2000) arXiv:hep-th/0002159; I. R. Klebanov and M. J. Strassler, "Supergravity and a confining gauge theory: Duality cascades and chiSB-resolution of naked singularities," JHEP 0008, 052 (2000) arXiv:hep-th/0007191. 
[28] N. Drukker, D. J. Gross and H. Ooguri, "Wilson loops and minimal surfaces," Phys. Rev. D 60, 125006 (1999) arXiv:hep-th/9904191.

[29] D. J. Gross and H. Ooguri, "Aspects of large N gauge theory dynamics as seen by string theory," Phys. Rev. D 58, 106002 (1998) arXiv:hep-th/9805129.

[30] K. Zarembo, "Wilson loop correlator in the AdS/CFT correspondence," Phys. Lett. B 459, 527 (1999) arXiv:hep-th/9904149]; P. Olesen and K. Zarembo, "Phase transition in Wilson loop correlator from AdS/CFT correspondence," arXiv:hep-th/0009210.

[31] H. Kim, D. K. Park, S. Tamarian and H. J. W. Muller-Kirsten, "Gross-Ooguri phase transition at zero and finite temperature: Two circular Wilson loop case," JHEP 0103, 003 (2001) arXiv:hep-th/0101235.

[32] N. Drukker and B. Fiol, "On the integrability of Wilson loops in AdS(5) x S**5: Some periodic ansatze," JHEP 0601, 056 (2006) arXiv:hep-th/0506058].

[33] C. Bachas, "Convexity Of The Quarkonium Potential," Phys. Rev. D 33, 2723 (1986).

[34] J. Greensite and P. Olesen, "Worldsheet fluctuations and the heavy quark potential in the AdS/CFT approach," JHEP 9904, 001 (1999) arXiv:hep-th/9901057.

[35] H. Dorn and V. D. Pershin, "Concavity of the Q anti-Q potential in N = 4 super YangMills gauge theory and AdS/CFT duality," Phys. Lett. B 461, 338 (1999) arXiv:hepth/9906073]. 